\title{
Estudo retrospectivo dos transplantes penetrantes de córnea da Santa Casa de Porto Alegre
}

\author{
Retrospective study of penetrating Keratoplasty at the Santa Casa of Porto Alegre
}

Caroline Fabris ${ }^{1}$
Zélia Maria $\quad$ S. $\quad$ Corrêa $^{2}$
Alexandre $\quad$ S. $\quad$ Marcon $^{3}$
Terla Nunes de $\quad$ Castro $^{3}$
Ítalo Mundialino $\quad$ Marcon $^{4}$
Cristiane $\quad$ Pawlowskit

Estudo realizado no Serviço de Oftalmologia da Santa Casa de de Porto Alegre (ISCMPA)/ Fundação Faculdade Federal de Ciências Médicas de Porto Alegre (FFFCMPA), Porto Alegre, RS

${ }^{1}$ Médica e ex-estagiária do Serviço de Oftalmologia da Santa Casa de Porto Alegre e Fundação Faculdade de Ciências Médicas de Porto Alegre, Porto Alegre, RS.

${ }^{2}$ Oftalmologista do Serviço de Oftalmologia da Santa Casa de Porto Alegre e Fundação Faculdade de Ciências Médicas de Porto Alegre, Porto Alegre, RS. Pósgraduanda (nível doutorado) Universidade Federal de São Paulo (UNIFESP-EPM).

${ }^{3}$ Oftalmologista do Setor de Córnea do Serviço de Oftalmologia da Santa Casa de Porto Alegre / Fundação Faculdade de Ciências Médicas de Porto Alegre

${ }^{4}$ Chefe do Departamento de Oftalmologia Serviço de Oftalmologia da Santa Casa de Porto Alegre e Professor Regente da Disciplina de Oftalmologia da Fundação Faculdade de Ciências Médicas de Porto Alegre, Porto Alegre, RS. Mestre e Doutor em Oftalmologia pela Universidade Federal de São Paulo.

${ }^{5}$ Residente do Serviço de Oftalmologia da Santa Casa de Porto Alegre e Fundação Faculdade de Ciências Médicas de Porto Alegre, Porto Alegre, RS

Endereço para correspondência: Dra. Caroline Fabris - R. Major Ernesto Vitrock, 67 - Centro Canoas (RS) CEP 92310-280. E-mail: Fabris@ca.conex.com.br

\section{RES UMO}

Objetivos: Traçar o perfil clínico e social dos pacientes submetidos a transplante de córnea e avaliar as principais indicações desses transplantes, tempo de espera para doação e os índices de sucesso, rejeição e falência dos transplantes penetrantes de córnea. Métodos: Foram analisados retrospectivamente os prontuários de 87 pacientes submetidos a transplante penetrante de córnea no Serviço de Oftalmologia da Santa Casa de Porto Alegre entre janeiro de 1990 e dezembro de 1998, totalizando 91 olhos. Destes, 56 pacientes foram chamados para avaliação clínica do enxerto durante o levantamento de dados para este estudo. Resultados: Considerando-se os 91 transplantes (87 pacientes), 53 (60,9\%) foram realizados em pacientes do sexo masculino e 34 (39\%), do sexo feminino. A idade variou de 3 a 89 anos, com média de 42,3 $\pm 19,8$ anos. As principais indicações para transplante de córnea foram ceratocone em $32(35 \%)$ olhos, ceratopatia bolhosa (pós-facectomia, distrofia de Fuchs e outros) em 24 $(26,4 \%)$, trauma mecânico em $9(9,9 \%)$, causas infecciosas em $9(9,9 \%)$, queimadura química em $2(2,2 \%)$, queimadura térmica em $1(1,1 \%)$ e outras causas em 14(15,4\%) olhos. O tempo médio de espera por doação de córnea foi de 17,3 \pm 9,9 meses. Do total de 91 olhos transplantados, $25(27,5 \%)$ apresentaram rejeição e 12 (13,2\%) olhos apresentaram falência do transplante. Conclusões: Este estudo mostrou a epidemiologia dos pacientes transplantados. A falta de dados seriados devido à alta precoce e perda de acompanhamento dos pacientes tornou impossível a análise e a comparação dos resultados pós-operatórios com outros estudos.

Descritores: Córnea/cirurgia; Transplante de córnea/etiologia; Transplante de córnea/ estatística e dados numéricos; Ceratoplastia penetrante; Sobrevivência de enxerto; Doadores de orgãos

INTRODUÇÃO

O primeiro transplante de córnea tecnicamente bem sucedido foi relatado por Power em 1878, mas após 20 dias houve perda da transparência do enxerto $^{(1)}$. Em 1905, Edward Zirm realizou um transplante de córnea que se manteve claro por mais de 1 ano $^{(1)}$.

O sucesso desse procedimento cirúrgico tem sido atribuído ao fato da córnea ser avascular e a privilégios imunológicos desta estrutura. Avanços na técnica cirúrgica, equipamento, materiais, métodos de preservação da córnea doadora e manejo pós-operatório também têm contribuído para o sucesso dos transplantes de córnea ${ }^{(2)}$.

O enxerto penetrante de córnea apresenta $90 \%$ de chance de se manter transparente ${ }^{(3)}$. Se este enxerto for realizado em um olho que apresenta 
rejeição prévia ou vascularização do estroma corneano, a chance de sucesso cai para $65 \%$ num intervalo de 3 anos $^{(4)}$.

O Serviço de Oftalmologia da Irmandade da Santa Casa de Porto Alegre (ISCMPA) realiza transplantes de córnea desde 1938. As doações de córnea tornaram-se mais freqüentes a partir do início da década de 90, motivadas, em parte, pela Central de Transplantes do Rio Grande do Sul, responsável pela captação e distribuição de orgãos doados neste estado. Em 1998 foram realizados 45 transplantes de córnea na Santa Casa de Porto Alegre.

Até o presente momento, pouco se conhece a respeito dos transplantes penetrantes de córnea realizados nesta instituição. Desta forma, este estudo, cuja importância é epidemiológica, tem como objetivos traçar o perfil clínico e social dos pacientes transplantados, avaliar as principais indicações dos transplantes, o tempo de espera para doação e os índices de sucesso, rejeição e falência dos transplantes penetrantes de córnea.

\section{MÉTODOS}

Foram analisados os prontuários de 87 pacientes (91 olhos) submetidos a transplante penetrante de córnea entre janeiro de 1990 e dezembro de 1998, de um total de 122 transplantes penetrantes de córnea realizados nesse período. Todos os transplantes foram realizados na Santa Casa de Misericórdia de Porto Alegre.

Os pacientes transplantados com endereço atualizado em seus prontuários (56 pacientes) foram contatados através de correspondência para reavaliação clínica de seu transplante de córnea. Trinta e um pacientes compareceram ao serviço e foram avaliados quanto aos principais sinais e sintomas, acuidade visual, e ocorrência de falência ou rejeição do transplante atual. Os resultados do exame foram anotados em um protocolo padronizado no qual constavam, além dos dados de identificação do paciente (raça, idade e sexo), campos para preenchimento dos ítens: procedência, acuidade visual prétransplante e pós-transplante (usando buraco estenopeico), indicação do transplante, tempo de espera por doação de córnea, doenças sistêmicas associadas e a aderência dos pacientes ao acompanhamento no serviço de oftalmologia.

Consideramos qualquer melhora da acuidade visual uma medida de sucesso terapêutico, sendo comparadas a acuidade visual pré e pós-transplante apenas dos pacientes que compareceram à consulta solicitada por correspondência.

Foi considerado insucesso terapêutico os botões corneanos com perda da transparência e conseqüente baixa da acuidade visual, causados por rejeição, falência do enxerto ou recorrência da doença ocular de base. Os critérios para classificar a rejeição do transplante foram ocorrência de linhas de rejeição epitelial ou endotelial, infiltrados epiteliais no enxerto, quantidade significativa de precipitados ceráticos, reação na câmara anterior ou aumento na espessura do estroma. Falência do transplante é definida como perda irreversível da transparência do centro do enxerto sem causa aparente ${ }^{(4)}$.

Os prontuários dos pacientes que não compareceram ao serviço para reavaliação ( 25 pacientes) e aqueles sem endere- ço atualizado em seus prontuários (31 pacientes) foram analisados da mesma forma que os pacientes reavaliados, exceto quanto à acuidade visual.

A análise dos dados obtidos é exposta neste trabalho através de médias, desvio padrão e porcentagens.

\section{RESULTADOS}

\section{Perfil dos pacientes}

Dos 87 pacientes estudados, 4 realizaram transplante bilateral, totalizando 91 olhos transplantados, excluindo-se os transplantes prévios. Foram realizados $53(60,9 \%)$ transplantes em pacientes do sexo masculino, e 34 (39\%) em pacientes do sexo feminino. A idade da amostra estudada variou de 3 a 89 anos, sendo a idade média de $42,3 \pm 19,8$ anos. A cor da pele mais freqüentemente observada foi a branca, com 74 pacientes $(89,2 \%)$, seguida da cor negra, com $9(10,8 \%)$. Quatro pacientes não apresentavam a cor da pele disponível no prontuário.

Apenas 17 pacientes (19,5\%), apresentavam doenças sistêmicas, sendo estas listadas em ordem de freqüência na tabela 1 .

A área de abrangência do serviço de transplantes de córnea está demonstrada de acordo com a procedência dos pacientes (Tabela 2). Considerando-se os pacientes trans-

\begin{tabular}{|c|c|c|}
\hline DOENÇAS SISTÊMICAS & $N^{\circ}$ DE PACIENTES & $\%$ \\
\hline Hipertensão arterial & 6 & 6,89 \\
\hline Diabetes Mellitus & 2 & 2,29 \\
\hline Diabetes e Hipertensão & 2 & 2,29 \\
\hline Hipertireoidismo & 2 & 2,29 \\
\hline Insuficiência renal crônica & 1 & 1,14 \\
\hline Artrose & 1 & 1,14 \\
\hline Bronquite & 1 & 1,14 \\
\hline Úlcera Péptica & 1 & 1,14 \\
\hline Carcinoma da próstata & 1 & 1,14 \\
\hline TOTAL & 17 & $19,5 \%$ \\
\hline \multicolumn{3}{|c|}{$\begin{array}{l}\text { Abreviaturas: } \mathrm{N}^{\circ} \text { de pacientes= número de pacientes com alguma doença } \\
\text { sistêmica, Total= total de pacientes com alguma patologia sistêmica na amostra } \\
\text { de } 87 \text { pacientes estudados }\end{array}$} \\
\hline
\end{tabular}

\begin{tabular}{|c|c|c|}
\hline REGIÕES & $N^{\circ}$ DE PACIENTES & $\%$ \\
\hline Porto Alegre & 36 & 41,4 \\
\hline Região Metropolitana (POA) & 22 & 25,3 \\
\hline Interior do RS & 26 & 29,9 \\
\hline Santa Catarina & 3 & 3,4 \\
\hline TOTAL & 87 & 100 \\
\hline \multicolumn{3}{|c|}{$\begin{array}{l}\text { Abreviaturas: } \mathrm{N}^{\circ} \text { de pacientes= número de pacientes provenientes de cada região, } \\
\text { Região Metropolitana }(\mathrm{POA})=\text { região metropolitana de Porto Alegre, Interior do RS= } \\
\text { interior do Rio Grande do Sul exceto área metropolitana de Porto Alegre }\end{array}$} \\
\hline
\end{tabular}


plantados estudados, $36(41,4 \%)$ pacientes eram procedentes de Porto Alegre, 22 (25,3\%) dos municípios da região metropolitana de Porto Alegre, $26(29,9 \%)$ do interior do Rio Grande do Sul e $3(3,4 \%)$ pacientes de Santa Catarina.

Do grupo de pacientes estudados, $44(50,6 \%)$ atualmente realizam acompanhamento no serviço de oftalmologia da Santa Casa de Porto Alegre e 43 (49,4\%) não.

\section{Indicações dos transplantes}

As principais indicações para transplante de córnea na Santa Casa de Porto Alegre foram ceratocone em 32 olhos (35\%), ceratopatia bolhosa em 24 olhos (26,4\%), trauma mecânico em 9 $(9,9 \%)$, causas infecciosas em $9(9,9 \%)$, queimadura química em $2(2,2 \%)$, queimadura térmica em $1(1,1 \%)$. Outras causas de indicação de transplante foram encontradas em 14 (15,4\%) olhos (tabela 3). Dentre os 24 olhos com ceratopatia bolhosa, 16 eram secundários à pseudofacia, 3 por afacia, 3 por distrofia de Fuchs e 2 prontuários não continham esta informação.

O tempo médio de espera por doação de córnea variou entre zero e 39 meses, com média de 17,3 $\pm 9,9$ meses.

\section{Sucesso dos transplantes de córnea}

Considerando os 31 pacientes submetidos ao transplante de córnea e avaliação oftalmológica na época deste estudo, 20 $(64,5 \%)$ apresentaram melhora da acuidade visual apesar de 4 destes pacientes apresentarem sinais de rejeição ativa. Dos 11 pacientes restantes que não apresentaram melhora da acuidade visual, 8 pacientes apresentaram sinais de rejeição no momento da consulta e os 3 restantes não apresentavam sinais de rejeição. Não houve falência de nenhum transplante examinado.

Os 56 pacientes que não realizaram avaliação oftalmológica durante o estudo, tiveram os dados quanto a rejeição e falência obtidos dos prontuários, entretanto, por ser irregular o período de "follow-up" dos pacientes, não foi possível uma organização cronológica deste evento.

Considerando os 91 olhos estudados, 25 olhos $(27,5 \%)$ apresentaram rejeição do transplante em algum momento, sendo que 1 destes evoluiu para atrofia bulbar (phithisis bulbi).

\begin{tabular}{|c|c|c|}
\hline INDICAÇÕES & № TRANSPLANTES & $\%$ \\
\hline Ceratocone & 32 & 35,1 \\
\hline Distrofia bolhosa* & 24 & 26,4 \\
\hline Trauma mecânico & 9 & 9,9 \\
\hline Causas infecciosas & 9 & 9,9 \\
\hline Queimadura química & 2 & 2,2 \\
\hline Queimadura térmica & 1 & 1,1 \\
\hline Outros & 14 & 15,4 \\
\hline TOTAL & 91 & 100 \\
\hline \multicolumn{3}{|c|}{$\begin{array}{l}\text { *Pós facectomia-pseudofacia }(n=16) \text {, devido à afacia }(n=3) \text {, Distrofia de Fuchs } \\
(n=3) \text {, prontuários sem etiologia da bolhosa }(n=2) \\
\text { Abreviaturas: Indicações=indicações de transplantes de córnea, } N^{\circ} \text { transplan- } \\
\text { tes= número de olhos transplantados com cada indicação, Outros=outras } \\
\text { causas como ceratites virais, úlceras de córnea, etc }\end{array}$} \\
\hline
\end{tabular}

Observou-se falência do transplante em 12 olhos $(13,2 \%)$. Quarenta e nove olhos $(53,8 \%)$ não apresentaram rejeição ou falência do transplante e, em 5 olhos $(5,5 \%)$, não foi possível obter tais informações no prontuário. Não foi possível avaliar a acuidade visual, porque em muitos prontuários revisados, esse dado estava incompleto. Os resultados sobre rejeição, falência e melhora terapêutica de todos os 91 olhos estudados encontram-se no gráfico 1 .

Os achados biomicroscópicos nos 31 pacientes examinados foram: hiperemia conjuntival presente em 16 olhos, opacidade de córnea em 15 olhos, neovasos de córnea em 14 olhos, edema das bordas do botão doador em 11 olhos, presença de precipitados ceráticos em 5 olhos e células na câmara anterior em 1 olho. Os sintomas referidos por este grupo de pacientes foram: sensação de corpo estranho em 15 pacientes, embaçamento visual em 14, fotofobia em 13 e dor em 7 pacientes.

O tempo médio de espera por doação de córnea variou

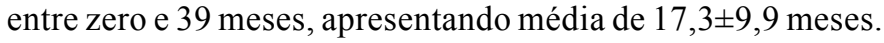
Recentemente, houve um aumento abrupto no número de transplantes penetrantes de córnea realizados nessa instituição (Gráfico 2).

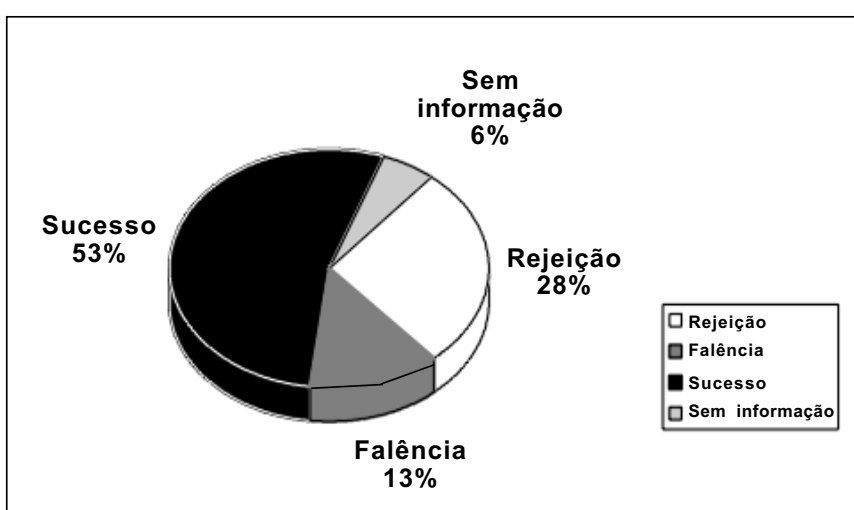

Sucesso $=$ porcentagem de transplantes de córnea bem sucedidos e com meios claros, Rejeição= porcentagem de transplantes com sinais de rejeição do botão doador, Falência= perda da transparência do botão doador, Sem informação= prontuários sem informação completa sobre o status do transplante

Gráfico 1 - Resultados dos transplantes de córnea realizados na Santa Casa de Porto Alegre no período de janeiro de 1990 a dezembro de 1998

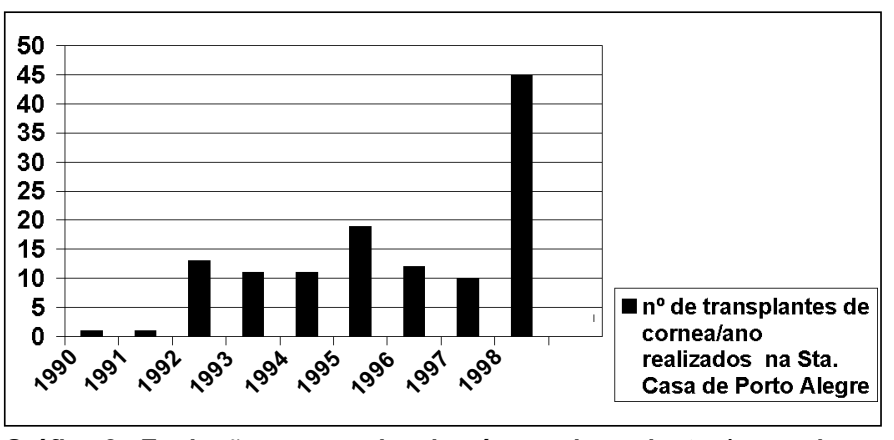

Gráfico 2 - Evolução progressiva do número de pacientes/ano submetidos a transplantes de córnea na Santa Casa de Porto Alegre entre janeiro de1990 e dezembro de 1998 


\section{DISCUSSÃO}

A idade da amostra estudada variou de 3 a 89 anos, comparável a outro estudo prospectivo publicado com 10 anos de seguimento, que apresentou faixa etária dos pacientes transplantados entre 3 e 94 anos $^{(5)}$.

Um estudo relatou que $18,7 \%$ dos pacientes submetidos a transplante de córnea em sua amostra eram portadores de doenças sistêmicas ${ }^{(6)}$. Este dado é semelhante aos apresentados neste estudo onde $17 \%$ dos pacientes apresentaram doenças sistêmicas associadas. A hipertensão arterial sistêmica foi a doença mais prevalente ${ }^{(6)} \mathrm{em}$ ambos estudos.

Em estudo realizado no Brasil, as principais indicações de 249 transplantes penetrantes de córnea foram: ceratocone em $21,7 \%$ dos pacientes, reoperação em $17,7 \%$, ceratite infecciosa em $14,9 \%$, trauma em $8,4 \%$, ceratopatia bolhosa pseudo-fácica em $8,0 \%$, distrofia não Fuchs em $6,4 \%$, ceratite ulcerativa não infecciosa em 4,0\%, distrofia de Fuchs em 3,6\%, Herpes em $3,6 \%$, vírus não Herpes em $2,4 \%$, ceratopatia bolhosa afácica em $2,0 \%$, queimadura em $1,2 \%$, entre outros ${ }^{(7)}$. Outro estudo realizado no país com 265 pacientes submetidos a transplante penetrante de córnea, as principais indicações foram: ceratocone em $24,9 \%$ dos casos, leucoma em $22,6 \%$, opacificação pós transplante em $20 \%$, ceratopatia bolhosa em $15,5 \%$, ceratites em $6,4 \%$ e distrofias em $5,3 \%$ dos casos $^{(8)}$. Outro estudo recentemente publicado apresenta como indicações mais freqüentes para transplante de córnea o ceratocone e a ceratopatia bolhosa ${ }^{(9)}$. Estes dados são semelhantes aos observados nesse estudo. Ao contrário da maioria dos estudos brasileiros, a Mayo Clinic apresenta como principal indicação de transplante de córnea a distrofia de Fuchs (27\%), seguida de ceratocone $(21 \%)^{(5)}$.

O recente aumento no número de transplantes penetrantes de córnea realizados nessa instituição (Gráfico 2) pode ser explicado pelo maior empenho dos responsáveis pela captação das córneas, aprimoramento técnico-burocrático, reestruturação do serviço de oftalmologia e aumento do número de doadores incentivado por campanhas institucionais. Entretanto, estes valores são pouco expressivos quando comparados a serviços maiores, localizados em grandes metrópoles ${ }^{(7,9)}$.

Após várias tentativas de análise de dados quanto à rejeição, falência e sucesso terapêutico, os autores notaram a impossibilidade desta análise para comparação com outros estudos, porque muitos pacientes não estão mais em acompanhamento com o serviço e seus prontuários não bastam como fonte de dados para a análise seriada dos desfechos quanto à rejeição e falência destes pacientes.

\section{CONCLUSÃO}

Este estudo tornou possível o conhecimento da epidemiologia dos nossos pacientes transplantados e demonstrou a importância de estudos de "follow-up" para a avaliação dos resultados deste procedimento. Atualmente, um projeto de pesquisa para avaliação prospectiva de pacientes submetidos a transplante de córnea na Santa Casa de Porto Alegre está em andamento, o que possibilitará a monitorização de dados importantes como critérios de rejeição, falência e sucesso terapêutico.

\section{ABS TRACT}

Purpose: To describe the clinical and social profile of recipient patients of transplanted corneas, and to evaluate main indications for corneal transplants, waiting time for donor tissue, and percentages of success, rejection and failure of penetrating keratoplasty. Methods: Retrospective chart review of 87 patients submitted to penetrating keratoplasty at the Santa Casa of Porto Alegre, between January, 1990 and December, 1998 totalling 91 eyes. Fifty-six of these patients were called at the time of data analysis, for a clinical evaluation of the graft. Results: Of the 91 keratoplasties in 87 patients, 53 $(60.9 \%)$ were performed in males and $34(39 \%)$ in females. The age of these patients varied between 3 and 89 years, with an average of $42.3 \pm 19.8$ years. The main indications for penetrating keratoplasty at this Ophthalmology Department was keratoconus in $32(35 \%)$ eyes, bullous keratopathy (after cataract extraction, Fuchs dystrophy, etc) in 24 (26.4\%), mechanical trauma in $9(9.9 \%)$, infection in $9(9.9 \%)$, chemical burn in $2(2.2 \%)$, thermal burn in $1(1.1 \%)$ and other causes in $14(15.6 \%)$ patients. The waiting time for donor tissue was $17.3 \pm 9.9$ months. Of the 91 studied keratoplasties, 25 (27.5\%) presented with rejection and $12(13.2 \%)$ patients presented corneal graft failure. Conclusions: This study showed the epidemiology of patients submitted to penetrating keratoplasties. It was not possible to analyze postoperative results and to compare them with other studies since there were no available data due to premature discharge or loss of follow-up of patients.

Keywords: Cornea/surgery; Corneal transplantation/etiology; Penetrating keratoplasty; Corneal transplantation/statistical and numerical data; Graft survival; Organ donors

\section{REFERÊNCIAS}

1. Marcon IM, Costa Gama AJ, Seminotti N, Marcon AS. Transplante de córnea. In: Neumann J, Abud Filho M, Garcia WD. Transplante de órgãos e tecidos. São Paulo: Sarvier; 1997. p. 336-52.

2. Kamp TM, Fink NE, Enger C, Maguire MG, Stark WJ, Stulting RD. Patientreported symptoms associated with graft reactions in high-risk patients in the collaborative corneal transplantation studies. Cornea 1995;14:43-8.

3. Price FW Jr., Whitson WE, Marks RG. Graft survival in four common groups of patients undergoing penetrating keratoplasty. Ophthalmology 1991; 98:322-8.

4. The Collaborative Corneal Transplantation Studies Research Group. Effectiveness of histocompatibility matching in high-risk corneal transplantation. The Collaborative Corneal Transplantation Studies (CCTS). [commented Arch Ophthalmol 1992;100:1517-8]. Arch Ophthalmol 1992;110:1392-403. 
5. Ing JJ, Ing HH, Nelson LR, Hodge DO, Bourne WM. Ten-year postoperative results of penetrating keratoplasty. Ophthalmology 1998;105:1855-65.

6. Vail A, Gore SM, Bradley BA, Easty DL, Roger CA, Armitate WJ. Clinical and surgical factors influencing corneal graft survival, visual acuity, and astigmatism. Corneal Transplant Follow-up Study Collaborators. Ophthalmology 1996;103:41-9.

7. Dantas MCN, Dantas PEC, Holzchuh N, Lui Neto A, Giovedi Filho R, Giovedi M. et al. Indicações de transplante penetrante de córnea: 1991-1995. Arq Bras Oftalmol 1998;61:26-33

8. Gonçalves EC, Trindade FC. Ceratoplastia penetrante: alterações nas indicacões, 1983-1992. Arq Bras Oftalmol 1994;57:274-7.

9. Chalita MRC, Diazgranados EBM, Sato EH, Branco DC, Freitas D. Rejeição corneana pós transplante de córnea: análise de dados do banco de olhos do Hospital São Paulo-Escola Paulista de Medicina. Arq Bras Oftalmol 2000;63:55-8.

\section{PROMOÇÃO EXCLUSIVA PARA SÓCIOS DO CBO}

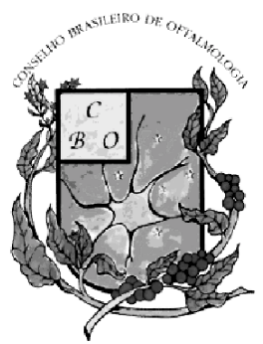

\section{Convênio CBO / Banco do Brasil: \\ a menor taxa de juros do mercado \\ TJPL + 4\% A.A.}

2. BANCO DO BRASIL

\section{Prezado Associado}

Conselho Brasileiro de Oftalmologia, em parceria com o Banco do Brasil, desenvolveu um produto para atender às suas necessidades.

Após longas negociações, entre as partes, chegamos ao que de melhor pode ser oferecido no mercado financeiro, em termos de crédito pessoal.

Trata-se de uma linha de crédito, destinada exclusivamente ao associado CBO, tanto pessoa física como jurídica, com valor limite de até $\mathrm{R} \$ 48.000,00$, com até 12 meses de carência, para início de pagamento do principal, e liquidação do financiamento (principal + juros) em até 73 meses.

Esta linha de crédito pode ser usada para:

- Equipamentos de uso profissional;

- Móveis e Computadores;

- Reformas.

Os juros aplicados serão de $4 \%$ a.a. (nominais), acrescidos da TJLP (taxa de Juros de longo prazo) vigente na época, e as garantias poderão ser negociadas entre várias opções, diretamente pelo interessado, junto à agência do Banco do Brasil, de seu domicílio.

Para informações, entre em contato com sua agência do Banco do Brasil, informando que esse Convênio refere-se ao LIC n. ${ }^{\circ}$ 2-15-12-302-1 do Programa Cooperfat. Maiores detalhes poderão ser obtidos pelo telefone (11) 5574-7441(Banco do Brasil - Agência EPM). Caso seja necessário alguma outra informação de responsabilidade do CBO, ligue (11) 3266-4000 (Sra. Tânia).

\section{CBO - Em defesa do exercício da Oftalmologia}

